\title{
Phonon Mediated Electron-Electron Scattering in Metals
}

\author{
V. Palenskis ${ }^{1}$, E. Žitkevičius ${ }^{2}$ \\ ${ }^{1}$ Physics Faculty of Vilnius University, Vilnius, Lithuania \\ ${ }^{2}$ Department of Electronic Systems, Vilnius Gediminas Technical University, Vilnius, Lithuania \\ Email: vilius.palenskis@ff.vu.lt
}

How to cite this paper: Palenskis, V. and Žitkevičius, E. (2018) Phonon Mediated Electron-Electron Scattering in Metals. World Journal of Condensed Matter Physics, 8, 115-129.

https://doi.org/10.4236/wjcmp.2018.83008

Received: July 30, 2018

Accepted: August 24, 2018

Published: August 27, 2018

Copyright (c) 2018 by authors and Scientific Research Publishing Inc. This work is licensed under the Creative Commons Attribution-NonCommercial International License (CC BY-NC 4.0). http://creativecommons.org/licenses/by-nc/4.0/ (c) (7) \& Open Access

\begin{abstract}
It is shown that the linear resistivity dependence on temperature for metals above the Debye's temperature mainly is caused by electron-electron scattering of randomly moving electrons. The electron mean free path in metals at this temperature range is in inverse proportion to the effective density of randomly moving electrons, i.e. it is in inverse proportion both to the temperature, and to the density-of-states at the Fermi surface. The general relationships for estimation of the average diffusion coefficient, the average velocity, mean free length and average relaxation time of randomly moving electrons at the Fermi surface at temperatures above the Debye's temperature are presented. The effective electron scattering cross-sections for different metals also are estimated. The calculation results of resistivity dependence on temperature in the range of temperature from $1 \mathrm{~K}$ to $900 \mathrm{~K}$ for $\mathrm{Au}, \mathrm{Cu}, \mathrm{Mo}$, and $\mathrm{Al}$ also are presented and compared with the experimental data. Additionally in temperature range from $1 \mathrm{~K}$ to $900 \mathrm{~K}$ for copper, the temperature dependences of the mean free path, average diffusion coefficient, average drift mobility, average Hall mobility, average relaxation time of randomly moving electrons, and their resultant phonon mediated scattering cross-section are presented.
\end{abstract}

\section{Keywords}

Effective Density of Randomly Moving Electrons, Electron-Electron Scattering, Electron Diffusion Coefficient, Electron Mean Free Path, Scattering Cross-Section

\section{Introduction}

The foundations of the electron theory of metals were laid on the preposition of free electrons which are assumed to be of the same order of valence electrons per 
unit volume. In order to obtain the correct order of the magnitude for conductivity and correct temperature variation of the mean free path of electrons at room temperature range, it was assumed that electron mean path is caused by thermal vibrations of lattice [1]. On the other hand, it is well known that electrons obey the Pauli principle and that electrons are described by the Fermi-Dirac statistics. The latter statistics let to explain the experimental results of the electron heat capacity of metals: Why metals and insulators have around the same heat capacity. The resolution of this paradox is one of the greatest successes of the Sommerfeld's model [2]. Considering this model the main conclusion has been done that only a small part of electrons with energy close to the Fermi level energy can randomly move, and that electrons which energy is well below the Fermi level cannot change their energy because all neighbor levels are occupied. The main weakness of the Sommerfeld's model is that the effective density of randomly moving electrons was not properly defined which can be scattered affected by external fields. So, the total density of valence electrons $n$ traditionally used for description of the electrical conductivity of the metals is unacceptable. Concerning that the Sommerfeld's model is based on the spherical Fermi surface, there also are uncertainties in determination of both the density-of-states (DOS) in conduction band at the Fermi energy $E_{\mathrm{F}}$, and the Fermi energy, because the Fermi surfaces for many of metals are not spherical [3]. The adequate definition for the effective density $n_{\text {eff }}$ of the randomly moving electrons in metals is presented in [4] [5].

This study mainly is addressed to using the stochastic description of the effective density of randomly moving electrons to investigation of the electron scattering mechanism at temperatures above the Debye's temperature. Additionally we estimated the resistivity dependence on temperature for $\mathrm{Au}, \mathrm{Cu}, \mathrm{Mo}$, and $\mathrm{Al}$ in the temperature range from $1 \mathrm{~K}$ to $900 \mathrm{~K}$. The mentioned problems are very important in solid state physics for relevant understanding and description of kinetic phenomena in metals, and semiconductors with highly degenerate electron gas.

\section{Results and Discussions}

\subsection{Basic Properties of Randomly Moving Electrons}

It is well known that the Fermi distribution function $f(E)$ for electrons is expressed as

$$
f(E)=1 /[1+\exp (E-\eta) / k T] .
$$

It specifies a probability that a level with the energy $E$ on average is occupied by an electron. Here, $E$ is the electron energy; $\eta$ is the chemical potential; $k$ is the Boltzmann's constant, and $T$ is the absolute temperature. Considering that the difference between the chemical potential and Fermi energy $E_{\mathrm{F}}$ is only about $0.01 \%$ for room temperature [2], further for calculation we use the Fermi distribution function in the following form:

$$
f(E)=1 /\left[1+\exp \left(E-E_{\mathrm{F}}\right) / k T\right] .
$$


Thus, the total density of the free valence electrons $n$ in conduction band is described by an integral on the density-of-states $g(E)$ as

$$
n=\int_{0}^{\infty} g(E) f(E) \mathrm{d} E .
$$

The electrical conductivity depends not only on the density-of-states $g(E)$ in conduction band and Fermi distribution function $f(E)$, but it also depends on the probability $f_{1}(E)=1-f(E)$ that any electron with the definite energy $E$ at a given temperature $T$ can be thermally scattered or can change his energy under influence of the external fields. Thus, the effective density of electrons $n_{\text {eff }}$ which take part in random motion and in conductivity is described by the probability

$$
h(E)=f(E)[1-f(E)] .
$$

Therefore, the effective density of randomly moving electrons $n_{\text {eff }}$ is described as [4] [5]

$$
n_{\text {eff }}=\int_{0}^{\infty} g(E) f(E)[1-f(E)] \mathrm{d} E=k T \int_{0}^{\infty} g(E)(-\partial f(E) / \partial E) \mathrm{d} E .
$$

From this expression follows that the term $(-\partial f(E) / \partial E)$ is the probability density function $p(E)$ that electron with energy $E$ is available to change his energy due to thermal and electrical influence:

$$
p(E)=(-\partial f(E) / \partial E)=h(E) / k T,
$$

and the integral probability distribution function is

$$
F(E)=\int_{0}^{E} p\left(E_{1}\right) \mathrm{d} E_{1}=1-f(E) .
$$

The functions $p(E)$ and $F(E)$ meet the requirements of the probability theory. The probability density dependence on energy of randomly moving electrons is presented in Figure 1. The area restricted by dash-line is equal to 1 , and the effective width of the probability density function in energy is $\Delta E_{\text {eff }}=4 k T$. Thus, the density of randomly moving electrons are described by probability density function $p(E)$, while the total density of the free electrons is constant quantity defined by the sum of valence electron density. The determination of the effective density of randomly moving electrons in homogeneous materials by Equation (5) is valid in all cases: with non-degenerate and with degenerate electron gas. For materials with non-degenerate electron gas the probability $[1-f(E)] \approx 1$ because $f(E) \ll 1$, and, therefore, all electrons in conduction band $n$ take part in random motion, and their density can be described as

$$
n_{\text {eff }}=n=\int_{0}^{\infty} g(E) f(E) \mathrm{d} E .
$$

This is the case when the classical statistics is applicable. In the case of highly degenerate electron gas, and considering that probability density function $(-\partial f(E) / \partial E)$ has a sharp maximum at $E=E_{\mathrm{F}}$, the Equation (5) can be presented in the following form: 


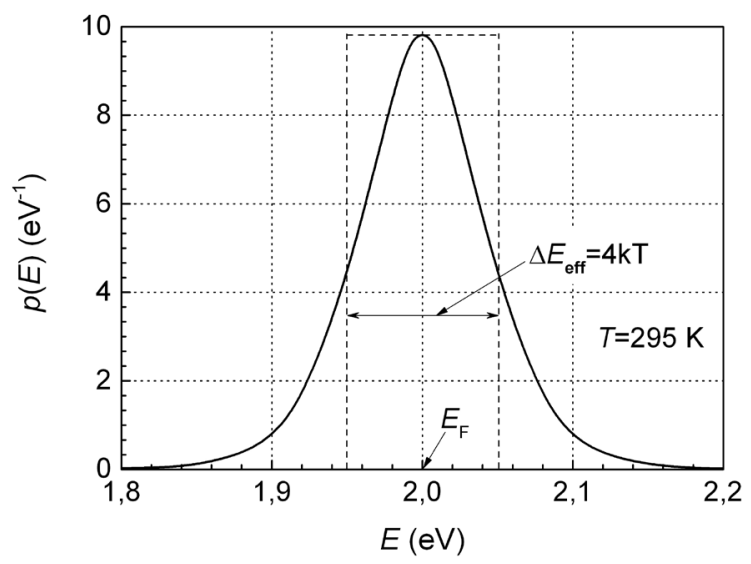

Figure 1 . The probability density $p(E)$ dependence on energy $E$ of randomly moving electrons near the Fermi energy $E_{\mathrm{F}}=2 \mathrm{eV}$ at $T=295 \mathrm{~K}$.

$$
n_{\text {eff }}=g\left(E_{\mathrm{F}}\right) k T \ll n,
$$

where $g\left(E_{\mathrm{F}}\right)=g(E)$ at $E=E_{\mathrm{F}}$. The density-of-states (DOS) at Fermi energy $g\left(E_{\mathrm{F}}\right)$ can be obtained from the experimental results of the electron heat capacity measurements [6]:

$$
c_{V}=\left(\pi^{2} / 3\right) g\left(E_{\mathrm{F}}\right) k^{2} T=\gamma T .
$$

Now we estimate the energy fluctuation of randomly moving electrons on the Fermi surface:

$$
\left\langle(E-\langle E\rangle)^{2}\right\rangle=\left\langle\left(E-E_{\mathrm{F}}\right)^{2}\right\rangle=\int_{0}^{\infty}\left(E-E_{\mathrm{F}}\right)^{2} g(E) f(E)[1-f(E)] \mathrm{d} E,
$$

or

$$
\begin{aligned}
\left\langle(E-\langle E\rangle)^{2}\right\rangle & =g\left(E_{\mathrm{F}}\right)(k T)^{3} \int_{0}^{\infty}\left(\varepsilon-\varepsilon_{F}\right)^{2} f(\varepsilon)[1-f(\varepsilon)] \mathrm{d} \varepsilon \\
& \cong 3.2899 g\left(E_{\mathrm{F}}\right)(k T)^{3} \cong \frac{\pi^{2}}{3} g\left(E_{\mathrm{F}}\right)(k T)^{3}
\end{aligned}
$$

From Equations (10) and (12) follows that

$$
\left\langle(E-\langle E\rangle)^{2}\right\rangle=k T^{2} c_{V}
$$

It is in accordance with the theory of energy fluctuation of free randomly moving particles [7]. This result shows that electron scattering is inelastic process.

The volume for one of randomly moving electron with the Fermi velocity for different metals can be estimated as:

$$
V_{\text {lel }}=1 / n_{\mathrm{eff}}=1 /\left[g\left(E_{\mathrm{F}}\right) k T\right],
$$

This volume increases with temperature decrease as $1 / T$.

For metals and other materials with highly degenerate electron gas the effective density of scattered electrons is described by Equation (9), and it is proportional to temperature. The DOS $g\left(E_{\mathrm{F}}\right)$ for various metals are presented in Figure 
2 (the values of the parameter $\gamma$ were taken from [6]). In the same figure there are presented the effective densities of randomly moving electrons

$n_{\text {eff }}=g\left(E_{\mathrm{F}}\right) k T$ at $T=295 \mathrm{~K}$. According to the Sommerfeld's model based on the spherical Fermi surface the DOS $g\left(E_{\mathrm{F}}\right)$ for metals is described as [2]

$$
g\left(E_{\mathrm{F}}\right)=\frac{m^{*}(3 n)^{1 / 3}}{\pi^{4 / 3} \hbar^{2}},
$$

where $m^{*}$ is the effective mass of the electron, and $\hbar=h / 2 \pi$ is the Plank's constant. This relation is presented in Figure 2 by solid line. This relation nearly describes the experimental results of $g\left(E_{\mathrm{F}}\right)$ for some of alkali and noble metals, but for other metals there are very large differences. Considering that energy structures of DOS for many metals are composite [3], there is no definite relation between the Fermi energy $E_{\mathrm{F}}$ and the total density of free electrons $n$, and also due to the same reason there is no definite relation between the Fermi energy $E_{\mathrm{F}}$ and the density-of-states at the Fermi surface.

\subsection{Conductivity and Diffusion Coefficient of Randomly Moving Electrons in Metals}

The electric conductivity $\sigma$ of the homogeneous materials and diffusion coefficient $D$ of randomly moving carriers can be described by such general expression [8] [9]:

$$
\sigma=q^{2} D\left(\frac{\partial n}{\partial \eta}\right)_{T} .
$$

After simple calculation ones obtain [4] [5]:

$$
\sigma=q^{2} D \int_{0}^{\infty} g(E) f(E)[1-f(E)] \mathrm{d} E=\frac{q^{2} D}{k T} n_{\text {eff }}=q \mu_{\text {drift }} n_{\text {eff }},
$$

where $\mu_{\text {drift }}$ is drift mobility of randomly moving charge carriers. These relations are valid for all homogeneous materials with a single type of charge carriers at any their degeneracy degree and it confirms that the electric conductivity for metals is determined by the effective density of randomly moving charge carriers, but not by the total density of free charge carriers. The distribution of experimental values of electric conductivity [6] for different metals on DOS at the Fermi surface at $T=295 \mathrm{~K}$ is presented in Figure 3(a).

For metals the Equation (17) can be rewritten as

$$
\sigma=q^{2} g\left(E_{\mathrm{F}}\right) D=(1 / 3) q^{2} g\left(E_{\mathrm{F}}\right) v_{\mathrm{F}}^{2} \tau_{\mathrm{F}}
$$

This expression is well known for metals, and it also can be obtained by solving Boltzmann's kinetic equation [10] [11]. The distribution of the diffusion coefficient $D$ of randomly moving electrons dependence on DOS for various metals is presented in Figure 3(b). It is interesting to note that electric conductivity for metals (Figure 3(a)) on average decreases with increasing of the density-of-states at the Fermi surface, i.e. with increasing of the effective density of randomly moving electrons. A spread of electric conductivity values in this fig- 
ure is completely caused by the spread of diffusion coefficient values as shown in Figure $3(\mathrm{~b})$. A tendency of conductivity decrease as $1 / g^{1 / 2}\left(E_{\mathrm{F}}\right)$ (Figure $3(\mathrm{a})$ ) is caused by diffusion coefficient tendency to decrease as $1 / g^{3 / 2}\left(E_{\mathrm{F}}\right)$ (Figure $3(\mathrm{~b})$ ).

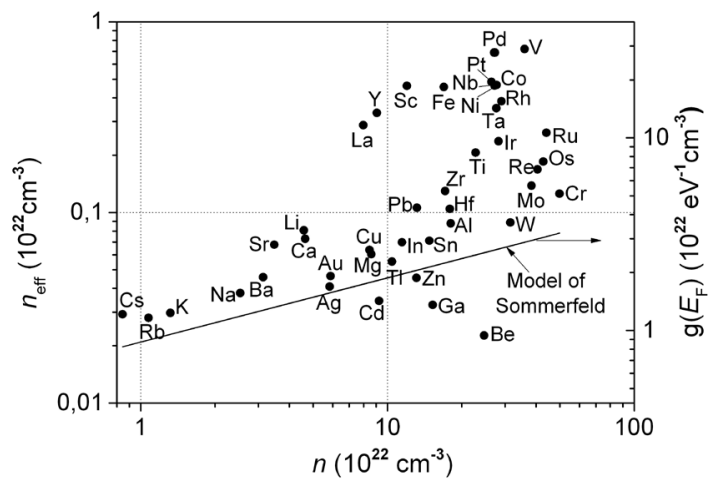

Figure 2. The effective density of randomly moving electrons $n_{\text {eff }}$ for different metals at $T=295 \mathrm{~K}$ (the left scale), and the density-of-states at Fermi surface $g\left(E_{\mathrm{F}}\right)$ (the right scale is selected in accordance to the left scale that positions of dots in the pattern were the same for both scales); the solid line represents the DOS $\mathrm{g}\left(E_{\mathrm{F}}\right)$ for Sommerfeld's model.

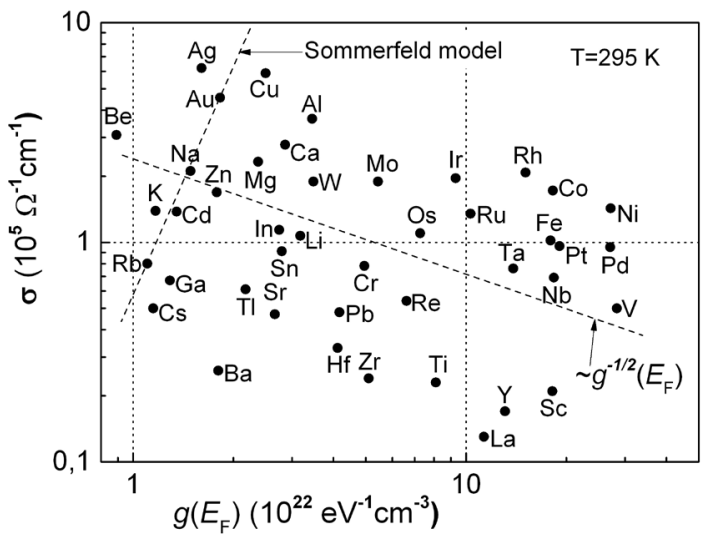

(a)

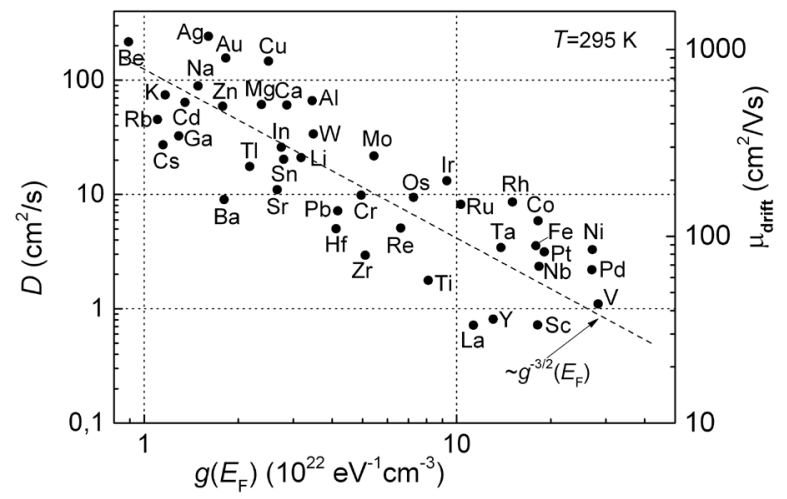

(b)

Figure 3. (a) Distribution of experimental values of electric conductivity $\sigma$ [6], and (b) diffusion coefficient $D$ of the randomly moving electrons for different metals on DOS at the Fermi surface at $T=295$ K. $D$ has been evaluated from Equation (18). 
From Equation (18) follows that diffusion coefficient of randomly moving electrons is related minimum with the five transport parameters of randomly moving electrons:

$$
D=\frac{\sigma}{q^{2} g\left(E_{\mathrm{F}}\right)}=\frac{1}{3} v_{\mathrm{F}}^{2} \tau_{\mathrm{F}}=\frac{1}{3} l_{\mathrm{F}} v_{\mathrm{F}},
$$

where $l_{\mathrm{F}}=v_{\mathrm{F}} \tau_{\mathrm{F}}$ is the mean free path of the electrons at the Fermi surface. What parameters mostly cause the spread of diffusion coefficient of electrons on density-of-states for metals at $E=E_{\mathrm{F}}$ ?

\subsection{The Mean Free Path of Randomly Moving Electrons in Metals at Temperatures above the Debye's Temperature}

The very important parameter characterizing the scattering mechanism of randomly moving charge carriers is their mean free path. In the case of electron-phonon scattering mechanism, the cross-section of scattering $\sigma_{\text {el-ph }}$ increases with temperature due to lattice ions vibration amplitude increasing [6] [8] [10]. It is interesting to find the relation of the mean free path of randomly moving electrons with their effective scattering cross-section $\sigma_{\text {eff. }}$ According to [6], the electron-electron scattering cross-section accounting the screening effect is about $10^{-15} \mathrm{~cm}^{2}$. All randomly moving electrons $n_{\text {eff }}$ with the average Fermi velocity $v_{\mathrm{F}}$ are scattered. Then the mean free path of scattered electrons in metals can be described as [6]

$$
l_{\mathrm{F}}=v_{\mathrm{F}} \tau_{\mathrm{F}}=1 /\left(\sigma_{\text {eff }} n_{\text {eff }}\right)=1 /\left[\sigma_{\text {eff }} g\left(E_{\mathrm{F}}\right) k T\right]
$$

and the average relaxation time as

$$
\tau_{\mathrm{F}}=1 /\left(\sigma_{\text {eff }} n_{\text {eff }} v_{\mathrm{F}}\right)=1 /\left(\sigma_{\text {eff }} g\left(E_{\mathrm{F}}\right) v_{\mathrm{F}} k T\right) .
$$

There it must be pointed that effective electron scattering cross-section $\sigma_{\text {eff }}$ doesn't depend on temperature at temperatures above the Debye's temperature. So, the statement that scattering cross-section at this temperature range is proportional to temperature contradicts to experimental resistivity data of metals. The Equation (20) directly shows that electron mean free path is in inverse proportion not only to temperature, but also to density-of-states at the Fermi surface. Besides the product $l_{\mathrm{F}} \sigma_{\text {eff }}=1 / n_{\text {eff }}=V_{\text {lel }}$ (here $V_{\text {lel }}$ is the volume for one randomly moving electron (Equation (15)). Considering that

$D=(1 / 3) l_{\mathrm{F}} v_{\mathrm{F}} \sim 1 / g^{3 / 2}\left(E_{\mathrm{F}}\right)$, and $l_{\mathrm{F}} \sim 1 / g\left(E_{\mathrm{F}}\right)$, it means that electron Fermi velocity $v_{\mathrm{F}} \sim 1 / g^{1 / 2}\left(E_{\mathrm{F}}\right)$, i.e. electron velocity at Fermi surface on average decreases with density-of-states increasing.

In paper [12], there are presented the calculation data of electron mean free path for large group of metals. It is interesting to find a relation between the mean free paths and diffusion coefficients of electrons. This relationship for a group of metals is presented in Figure 4. The relationship between the mean free path and the diffusion coefficient of randomly moving electrons in metals in the linear resistivity dependence on temperature range can be approximated as 


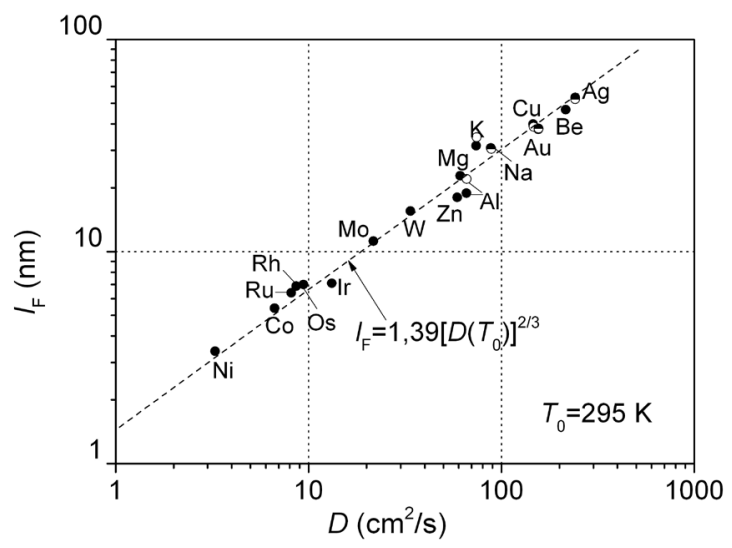

Figure 4. Relationship between the mean free path $I_{\mathrm{F}}$ and the diffusion coefficient $D$ of electrons for a group metals at room temperature $\left(T_{0}=295 \mathrm{~K} ; \bullet-\right.$ solid dots $I_{\mathrm{F}}$ from [12]; o- open dots $I_{\mathrm{F}}$ from dimension effect [13]; half filled dots - both data coincide).

$$
l_{\mathrm{F}}(T)=1.39\left(D\left(T_{0}\right)\right)^{2 / 3}\left(T_{0} / T\right)
$$

here $l_{\mathrm{F}}$ is in $\mathrm{nm}$, and $D\left(T_{0}\right)$ is in $\mathrm{cm}^{2} / \mathrm{s}$ at $T_{0}=295 \mathrm{~K}$. A small spread of the data in Figure 4 from Equation (22) may be originated from some uncertainty of estimation of the mean free path values due to a very complicate structure of the Fermi surfaces especially of the transient group metals. Some different values of the electron mean free path were obtained by other authors [14] [15]: the estimated $l_{\mathrm{F}}$ for $\mathrm{Cu}$ is $55 \mathrm{~nm}$, for $\mathrm{Al}$ is $22 \mathrm{~nm}$, and for $\mathrm{W}$ is $19.1 \mathrm{~nm}$, while for the same metals in the paper [12], respectively, there are such data: $39.9 \mathrm{~nm}, 18.9$ $\mathrm{nm}$, and $15.5 \mathrm{~nm}$.

From Equation (20) the effective electron-electron scattering cross-section can be estimated as

$$
\sigma_{\text {eff }}=1 /\left(l_{\mathrm{F}} n_{\text {eff }}\right)=1 /\left[l_{\mathrm{F}} g\left(E_{\mathrm{F}}\right) k T\right] .
$$

The effective electron scattering cross-sections are distributed in the range (0.40 - 3.6) $10^{-15} \mathrm{~cm}^{2}$, and they do not depend on temperature for that temperature range where the resistivity is proportional to temperature $T$. The effective electron-electron cross-section for every metal has a particular value, which depends on the Fermi surface structure. Considering that mean free path data for different metals are different due to specific effective electron scattering cross-section values, the spread of those values causes also the spread of both the conductivity (Figure 3(a)) and the diffusion coefficient (Figure 3(b)) values. From obtained results follows that the resistivity linear dependence on temperature above the Debye temperature $\Theta_{D}$ is caused by the effective randomly moving charge carrier density dependence on temperature.

From Equations (19)-(22) it is possible to design the following important pattern (Figure 5) where there are shown the relationships of the mean free path, average velocity, and the average relaxation time of the randomly moving electrons with their diffusion coefficient for different metals at temperature $295 \mathrm{~K}$. The average velocity of electrons at the Fermi surface can be described as 


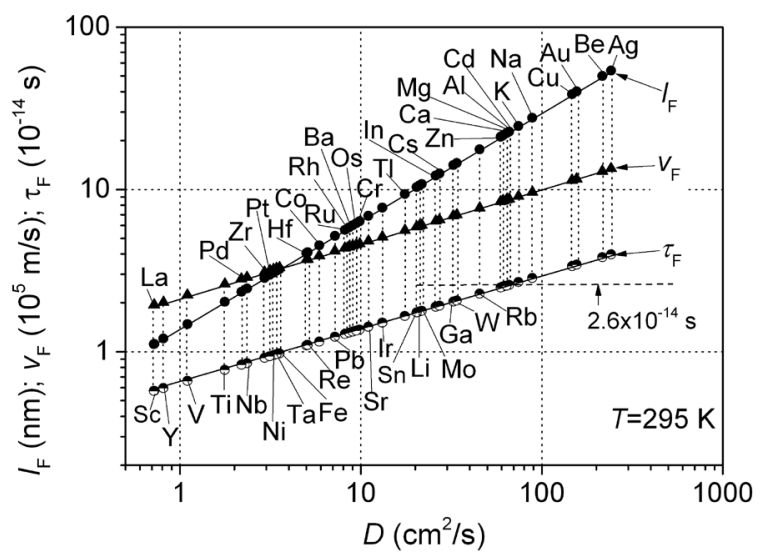

Figure 5. Relationships of the mean free path $l_{F}$ average velocity $v_{\mathrm{F}}$, and average relaxation time $\tau_{\mathrm{F}}$ of randomly moving charge carriers with their diffusion coefficient for different metals at $T=295 \mathrm{~K}$. The data dots for the same metals are connected with vertical dash lines.

$$
v_{\mathrm{F}}=2.165\left(D\left(T_{0}\right)\right)^{1 / 3}
$$

where $V_{\mathrm{F}}$ is in units $10^{5} \mathrm{~m} / \mathrm{s}$ and does not depend on temperature, $D\left(T_{0}\right)$ is in $\mathrm{cm}^{2} / \mathrm{s}$ at $T_{0}=295 \mathrm{~K}$, and similarly the average relaxation time of electrons at the Fermi surface in the linear resistivity dependence on temperature range as

$$
\tau_{\mathrm{F}}(T)=0.64\left(D\left(T_{0}\right)\right)^{1 / 3}\left(T_{0} / T\right),
$$

where $\tau_{\mathrm{F}}$ is in units $10^{-14} \mathrm{~s}$, and $D\left(T_{0}\right)$ in $\mathrm{cm}^{2} / \mathrm{s}$ at $T_{0}=295 \mathrm{~K}$.

There must be pointed that statement that average relaxation time for all metals in the range of the linear resistivity dependence on temperature can be expressed as $\tau_{\mathrm{F}} \cong \hbar / k T$ [16] is not right, because the average relaxation times for different metals at room temperature are in the range (0.6 - 4) $10^{-14} \mathrm{~s}$ (Figure 5), while this value according to [16] at $T=295 \mathrm{~K}$ must be about $2.6 \times 10^{-14} \mathrm{~s}$ (horizontal dash line in Figure 5).

In the case of electron-phonon scattering the cross-section must increase with temperature increasing [6], and it would originate the resistivity dependence on temperature as $T^{2}$ in the range of temperatures above the Debye's temperature, but it does not take place. Heretofore the linear resistivity dependence on temperature was explained by electron-phonon scattering keeping that all valence electrons take part in current transport, but it is inconsistent with the Pauli's principle and Fermi distribution function.

Accounting the real effective density of randomly moving electrons let us to conclude that electron-electron scattering is the main mechanism that causes the linear resistivity dependence on temperature of metals above the Debye's temperature.

\subsection{The Phonon-Mediated Correction to the Effective Electron-Electron Scattering Cross-Section}

It was shown that the effective electron scattering cross-section $\sigma_{\text {eff }}$ doesn't de- 
pend on temperature at temperatures above the Debye temperature, but also it was shown that average electron relaxation time at Fermi surface $\tau_{\mathrm{F}} \sim 1 / T$, and mean free path $l_{\mathrm{F}}=\mathrm{v}_{\mathrm{F}} \tau_{\mathrm{F}} \sim 1 / T$. Considering that at temperatures below the Debye's temperature the resistivity decreases with temperature more steeply then $1 / T$, there arise the question: What is the role of phonons? In Figure 6(a) there are presented the resistivity of $\mathrm{Au}, \mathrm{Cu}, \mathrm{Mo}$, and $\mathrm{Al}$ experimental dependences on temperature [17]. What effects cause such steep resistivity decreasing with temperature in temperature range below the Debye's temperature $\Theta$ ?

The independent resistivity part on temperature below $10 \mathrm{~K}$ is the residual electric resistivity due to scattering of electrons from chemical and structural imperfections in the investigated samples.

It seems that effective electron scattering cross-section depends on the exchange of thermal energies of lattice and randomly moving electrons, i.e. depends on the ratio of the thermal energy of the phonon to thermal energy of the electron. Though the kinetic energy of randomly moving electron is equal to $E_{\mathrm{k} 1}=m v_{\mathrm{F}}^{2} / 2$, but its thermal energy is only

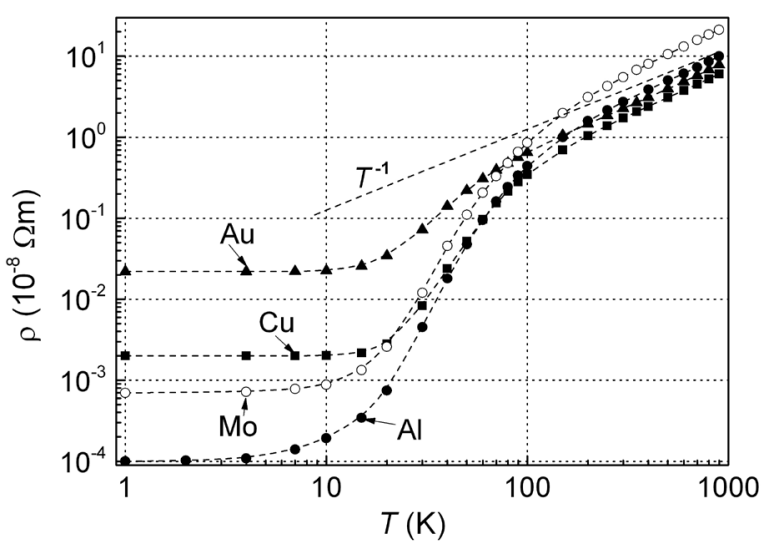

(a)

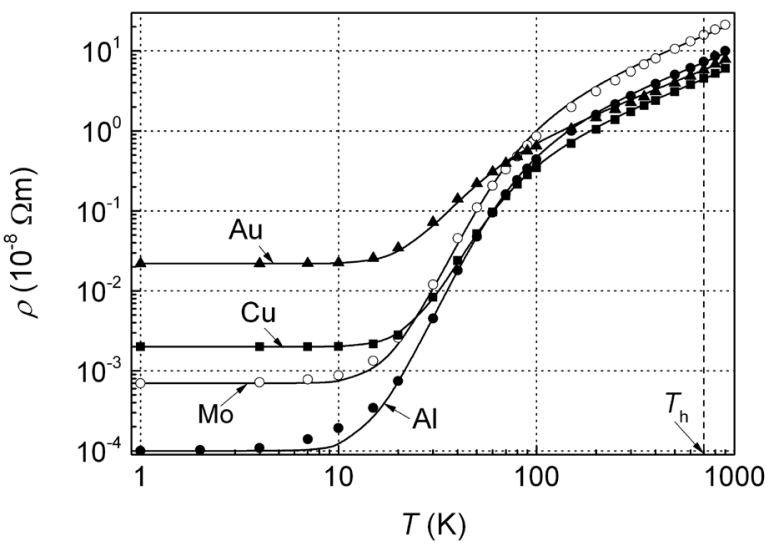

(b)

Figure 6. (a) Resistivity $\rho$ dependence on temperature $T$ for $\mathrm{Au}, \mathrm{Cu}, \mathrm{Mo}$ and $\mathrm{Al}$ : (a) dots are experimental data taken from [17], dash lines are used only for connections between dots; (b) solid lines are the calculation results from Equation (32); $T_{\mathrm{h}}=700 \mathrm{~K}$.; the other parameters used for calculations are listed in Table 1. 
Table 1. The parameters used for calculation $\mathrm{Au}, \mathrm{Cu}, \mathrm{Mo}$, and $\mathrm{Al}$ resistivity dependencies on temperature.

\begin{tabular}{ccccccc}
\hline Metal & $\begin{array}{c}\rho_{0}, 10^{-8} \Omega \mathrm{m} \\
\text { at } T=1 \mathrm{~K}\end{array}$ & $\begin{array}{c}\rho, 10^{-8} \Omega \mathrm{m} \\
\text { at } T_{\mathrm{h}}=700 \mathrm{~K}\end{array}$ & $V_{F}, 10^{6} \mathrm{~m} / \mathrm{s}$ & $\begin{array}{c}\Theta, \mathrm{K} \text { used } \\
\text { in this work }\end{array}$ & $\begin{array}{c}\Theta, \mathrm{K} \text { cited } \\
\text { in references }\end{array}$ & $\begin{array}{c}\sigma_{\text {eff, }} \\
10^{-15} \mathrm{~cm}^{2}\end{array}$ \\
\hline $\mathrm{Au}$ & 0.0220 & 5.816 & 1.16 & 200 & $165[7] ; 185[18]$ & 0.53 \\
$\mathrm{Cu}$ & 0.0020 & 4.514 & 1.14 & 343 & $310[18] ; 343[7]$ & 0.40 \\
$\mathrm{Mo}$ & 0.00070 & 15.81 & 0.603 & 410 & $375[18] ; 450[7]$ & 0.66 \\
$\mathrm{Al}$ & 0.00010 & 7.322 & 0.874 & 410 & $380[18] ; 428[7]$ & 0.50 \\
\hline
\end{tabular}

Note: the resistivities $\rho_{0}$ at $T=1 \mathrm{~K}$, and $\rho$ at $T_{\mathrm{h}}=700 \mathrm{~K}$ are taken from [17]; $\nu_{\mathrm{F}}$ is calculated from Equation (24); $\sigma_{\text {eff }}$ is calculated from Equation (23).

$$
E_{\mathrm{e} 1}=\left(\pi^{2} / 6\right)(k T)^{2} g\left(E_{\mathrm{F}}\right) / n_{\mathrm{eff}}=\left(\pi^{2} / 6\right)(k T) \approx 1.64 k T .
$$

The average phonon thermal energy at temperatures over Debye's temperature $\Theta$ is about $3 k T$ [7] [10] because all lattice waves are excited, but in temperature range below temperature $\Theta$ only the lattice waves with low frequencies are excited. Accounting that electron scattering process is inelastic, i.e. accounting the electron energy fluctuations, which cause the excitation and annihilation of phonons, the average phonon thermal energy can be described as [10]:

$$
E_{\mathrm{ph} 1}=3 k T\left(\frac{T}{\Theta}\right)^{4} \int_{0}^{\Theta / T} \frac{4 x^{5}}{\left(\mathrm{e}^{x}-1\right)\left(1-\mathrm{e}^{-x}\right)} \mathrm{d} x .
$$

Thus, the ratio of the average thermal energies between phonon to electron can be described as

$$
\frac{E_{\mathrm{ph} 1}}{E_{\mathrm{e} 1}}=\frac{3 k T}{1.64 k T}\left(\frac{T}{\Theta}\right)^{4} \int_{0}^{\Theta / T} \frac{4 x^{5}}{\left(\mathrm{e}^{x}-1\right)\left(1-\mathrm{e}^{-x}\right)} \mathrm{d} x \approx 1.83 \eta(T / \Theta),
$$

where

$$
\eta(T / \Theta)=\left(\frac{T}{\Theta}\right)^{4} \int_{0}^{\Theta / T} \frac{4 x^{5}}{\left(\mathrm{e}^{x}-1\right)\left(1-\mathrm{e}^{-x}\right)} \mathrm{d} x
$$

is the phonon mediation factor. So, the phonon mediated resultant electron scattering cross-section $\sigma_{\text {res }}$ can be described as

$$
\sigma_{\text {res }}=\sigma_{\text {eff }} \eta(T / \Theta) \text {. }
$$

here the quantity $\sigma_{\text {eff }}$ accounts the constant multiplier 1.83 , because at $T \gg \Theta$ from Equation (21) we obtain the quantity $\sigma_{\text {eff }}$ independent on $T$. The resultant average relaxation time $\tau_{\text {res }}$ is described as

$$
\frac{1}{\tau_{\text {res }}}=\frac{1}{\tau_{\text {eff }}}+\frac{1}{\tau_{\text {imp }}},
$$

where $\tau_{\text {eff }}=1 /\left(\sigma_{\text {res }} n_{\text {eff }} v_{\mathrm{F}}\right)=1 /\left(\sigma_{\text {res }} g\left(E_{\mathrm{F}}\right) v_{\mathrm{F}} k T\right)$, and $\tau_{\text {imp }}=1 /\left(\sigma_{\text {imp }} N_{\text {imp }} v_{\mathrm{F}}\right) ; \sigma_{\text {imp }}$ is the average cross-section of the impurity defect, $N_{\mathrm{imp}}$ is the impurity density.

The metal resistivity in the overall temperature range can be described as

$$
\rho_{\text {res }}=\rho_{0}+\rho\left(T_{\mathrm{h}}\right) \cdot\left(T / T_{\mathrm{h}}\right) \cdot \eta(T / \Theta),
$$




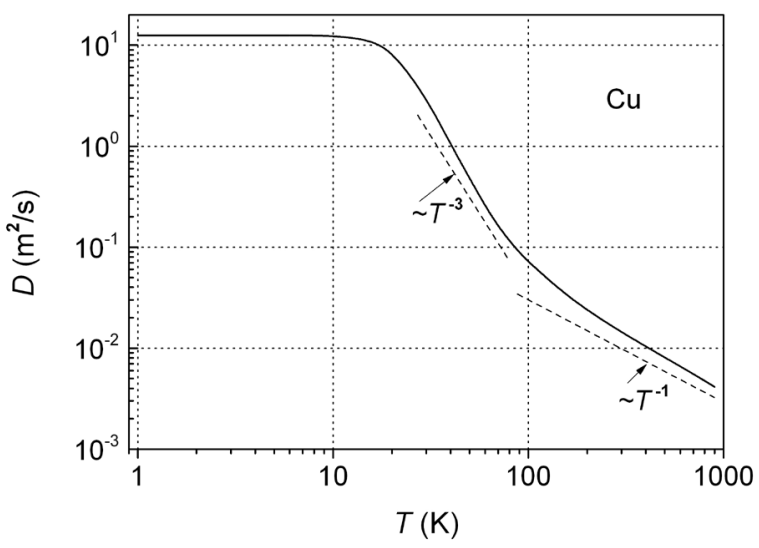

(a)

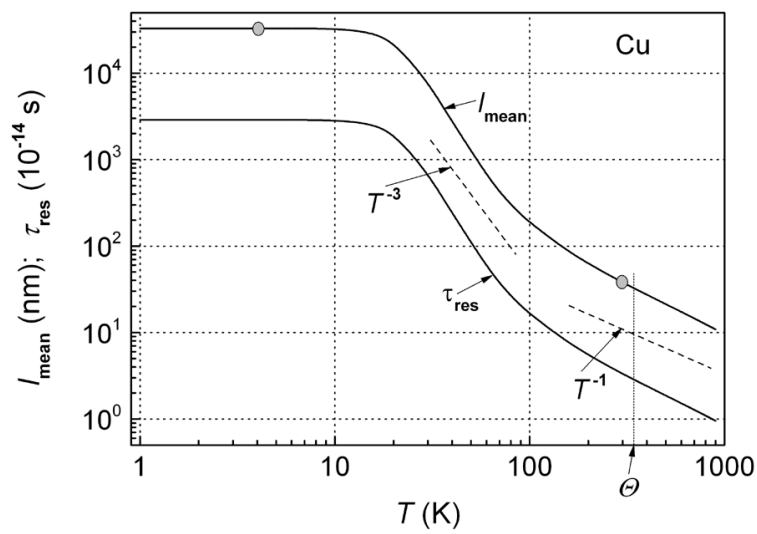

(b)

Figure 7. (a) The diffusion coefficient $D$ of randomly moving electrons dependency on temperature $T$ for $\mathrm{Cu}$; (b) the comparison of the mean free path $I_{\text {mean }}$ and the resultant electron relaxation time $\tau_{\text {res }}$ dependencies on temperature $T$ for $\mathrm{Cu}$; dots $I_{\text {mean }}$ are estimated from dimension effect [13].

where $T_{\mathrm{h}} \gg \Theta$ is the reference temperature in the high linearity range of the resistivity dependence on temperature (here for calculation we take $T_{\mathrm{h}}=700 \mathrm{~K}$ ). The resistivity dependence on temperature for $\mathrm{Au}, \mathrm{Cu}, \mathrm{Mo}$ and $\mathrm{Al}$ are presented in Figure 6(a). The parameters used for calculations are listed in Table 1. The Debye's temperature $\Theta$ has been chosen to the best agreement between calculation data from Equation (32) and experimental dots (Figure 6(b)). It is seen that calculation data sufficiently good agree with experimental results. A small disagreement between calculation and experimental data in particular temperature ranges may be is due to that Debye temperature can change with temperature [7] [18]. Here the calculations have been performed by using constant quantity $\Theta$, which values for calculated resistivities of a few metals are presented in the Table 1.

Considering that calculation data by using Equation (32) very well agree to the measurement results for $\mathrm{Cu}$ (Figure 6(b)), further we present the basic kinetic parameters for copper in temperature range from $1 \mathrm{~K}$ to $900 \mathrm{~K}$. In Figure 7(a) there is presented the diffusion coefficient of the randomly moving electrons 


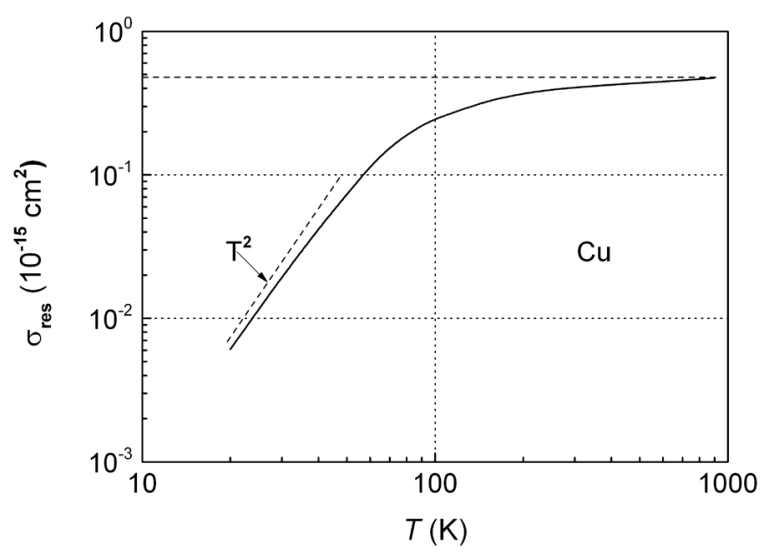

(a)

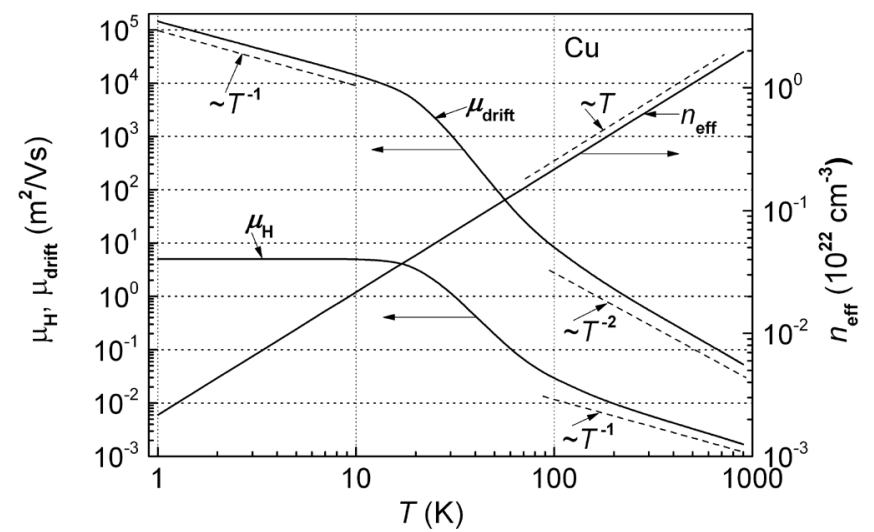

(b)

Figure 8. (a) The phonon mediated resultant electron-electron scattering cross-section $\sigma_{\text {res }}$ dependency on temperature for $\mathrm{Cu}$; (b) the effective density $n_{\text {eff }}$ drift mobility $\mu_{\text {drift }}$, and Hall mobility $\mu_{\mathrm{H}}$ of randomly moving electrons dependencies on temperature for $\mathrm{Cu}$.

dependency on temperature for $\mathrm{Cu}$. The diffusion coefficient has been evaluated from Equation (18). The comparison of the mean free path $l_{\text {mean }}$ and the resultant electron relaxation time $\tau_{\text {res }}$ of the randomly moving electrons dependencies on temperature for $\mathrm{Cu}$ in temperature range from $1 \mathrm{~K}$ to $900 \mathrm{~K}$ is presented in Figure $7(\mathrm{~b})$. The mean free path $I_{\text {mean }}$ was evaluated from relation $\mathrm{D}=(1 / 3) I_{\text {mean }} \mathrm{v}_{\mathrm{F}}$, and the resultant electron relaxation time $\tau_{\text {res }}$ was evaluated as $\tau_{\text {res }}=I_{\text {mean }} / V_{\mathrm{F}}$.

The resultant phonon mediated electron-electron scattering cross-section $\sigma_{\text {res }}$ is shown in Figure 8(a) at temperature higher than $20 \mathrm{~K}$, because at temperatures lower than $20 \mathrm{~K}$ electron scattering cross-section is determined by the neutral impurity atom cross-section. For the most of metals the cross-section is in the range $(0.5-1.2) \cdot 10^{-15} \mathrm{~cm}^{2}$ [18] (here for estimation of impurity density $N_{\text {imp }}$ we take $\sigma_{\text {imp }} \approx 0.7 \cdot 10^{-15} \mathrm{~cm}^{2}$ ). The effective density $n_{\text {eff }}$ drift mobility $\mu_{\text {drift, }}$ and Hall mobility $\mu_{\mathrm{H}}$ of randomly moving electrons dependencies on temperature from $1 \mathrm{~K}$ to $900 \mathrm{~K}$ are presented in Figure 8 (b). It is interesting to note that at temperatures over the Debye temperature the Hall mobility changes as $1 / T$, but drift mobility changes as $1 / T^{2}$, and at temperature below $20 \mathrm{~K}$ the Hall mobility is constant, but drift mobility changes as $1 / T$. Thus, considering that densi- 
ty-of-states and velocity of electrons at Fermi surface don't depend on temperature, it is easy from resistivity (conductivity) data to find the basic electron transport characteristics for metals.

\section{Conclusions}

The investigation results of the fundamental transport characteristics of free electrons on the base of the effective density of randomly moving electrons in metals have been presented. It let to estimate the real average characteristics of randomly moving electrons in metals, such as electron diffusion coefficient, drift mobility, free path, velocity, relaxation time, and to show that these quantities in homogeneous metals above the Debye temperature mainly are due to electron-electron scattering. It is shown that the effective electron scattering cross-section does not depend on temperature in the linear resistivity dependence on temperature range.

The calculation results of resistivity dependence on temperature in the range of temperature from $1 \mathrm{~K}$ to $900 \mathrm{~K}$ for $\mathrm{Au}, \mathrm{Cu}, \mathrm{Mo}$, and $\mathrm{Al}$ are presented and compared with the experimental data. Additionally for $\mathrm{Cu}$ at first time the mean free path, average diffusion coefficient, average drift mobility, average relaxation time of randomly moving electrons dependences on temperature from $1 \mathrm{~K}$ to $900 \mathrm{~K}$ are presented.

\section{Conflicts of Interest}

The authors declare no conflicts of interest regarding the publication of this paper.

\section{References}

[1] Sondheimer, E.H. (2001) The Mean Free Path of Electrons in Metals. Advances in Physics, 50, 499-537. https://doi.org/10.1080/00018730110102187

[2] Ashcroft, N.W. and Mermin, N.D. (1976) Solid State Physics (Ch. 2). Holt, Rinehart and Winston, New York, Chicago.

[3] Cracknell, A.P. and Wong, K.C. (1973) The Fermi Surfaces: Its Concept, Determination, and Use in the Physics of Metals. Clarendon Press, Oxford.

[4] Palenskis, V. (2013) Drift Mobility, Diffusion Coefficient of Randomly Moving Charge Carriers in Metals and Other Materials with Degenerate Electron Gas. World Journal of Condensed Matter Physics, 3, 73-81.

[5] Palenskis, V. (2014) The Effective Density of Randomly Moving Electrons and Related Characteristics of Materials with Degenerate Electron Gas. AIP Advances, 4, 047119-1(9).

[6] Kittel, Ch. (1976) Introduction to Solid States Solids. John Wiley and Sons, Inc., New York, London.

[7] Kittel, Ch. (1969) Thermal Physics. John Wiley and Sons, Inc., New York.

[8] Bonch-Brujevich, V.L. and Kalashnikov, S.G. (1990) The Physics of Semiconductors. Nauka Press, Moscow.

[9] Dugdale, J.S. (2010) The Electrical Properties of Disordered Metals. Cambridge 
University Press Cambridge.

[10] Ziman, J.M. (1972) Principles of the Theory of Solids. Cambridge University Press, Cambridge. https://doi.org/10.1017/CBO9781139644075

[11] Abrikosov, A.A. (1987) Principles of the Theory of Metals. Nauka Press, Moscow.

[12] Gall, D. (2016) Electron Mean Free Path in Elemental Metals. Journal of Applied Physics, 119, 085101-1(5).

[13] Chopra, K.L. (1979) Thin Film Phenomena. R. E. Krieger Publ. Comp., Science, Malabar.

[14] Hanaoka, Y., Hinode, K., Takeda, K. and Kodama, G. (2002) Increase in Electrical Resistivity of Copper and Aluminum Fine Lines. Materials Transactions, 43, 1621-1623. https://doi.org/10.2320/matertrans.43.1621

[15] Choi, D., Kim, Ch.S., Naveh, D., Waren, A.P., Toney, M.F., Coffey, K.R. and Barmak, K. (2012) Electron Mean Free Path of Tungsten and the Electrical Resistivity of Epitaxial (110) Tungsten Films. Physical Review B, 86, 045432-1(5).

[16] Devillers, M.A.C. (1984) Lifetime of Electrons in Metals at Room Temperature. Solid State Communications, 49, 1019-1022.

https://doi.org/10.1016/0038-1098(84)90413-7

[17] http://www.knowledgedoor.com/2/elements_handbook/electrical_resistivity.html

[18] Schulze, G.E.R. (1967) Metallphysik. Akdemie-Verlage, Berlin. 